\author{
Francesco Martinello
}

\title{
BARE PARTICULARS: SOME REMARKS
}

Abstract. The paper shows and discusses three of Bergmann's main reasons for acknowledging the ontological category of bare particulars. The first motivation is recognized in his agreement with the principle of acquaintance, according to which the simplest entities of ontological analysis must be presented to us directly. Since it seems very hard to maintain that we are acquainted with bare entities rather than with something qualitied, this argument turns out to be not very compelling. The second ground for admitting bareness is indeed that it seems to be an easy way to solve the problem of individuation. There are, however, some classical paradoxes of identity, which cannot be adequately handled with the theory of bare particulars. Finally, the strongest claim in support of bare particulars is seen in the ideal language method, which has been employed by Bergmann since his earliest works, and that has never been completely dismissed by him.

In this paper my concern is to make some remarks about the reasons which may lead a philosopher to include in his ontology such odd entities as bare particulars. Since Gustav Bergmann was one of the most important supporters of this ontological kind, my remarks will stand on the background of his works. I singled out in Bergmann's writings three reasons for acknowledging the ontological kind of bare particulars; each of them relates this notion to other important philosophical topics. I recognized three reasons for acknowledging bare particulars, but this obviously does not mean that they are the unique speculative motivations one could discover. Neither I want to hold that further approaches cannot be found in Bergmann's works. Quite to the contrary, I am confident that both the denial of internal relations and the topic of awareness are connected with the issue of bare particulars. Maybe they will be the subject of another paper of mine.

\section{Bare particulars and acquaintance}

The first couple of notions I would like to look at is bare particulars and acquaintance. It has been debated for a long time if an ontologist must agree to bare particulars on the ground of the so called Principle of Ac- 
quaintance $^{1}$. The basic idea is the following: we know that something exists because we are acquainted with it. This suggestion has been taken to have at least two meanings. The first is that, in order to regard something as the simple entities of ontological analysis, one must be presented with them. The second is that one must be acquainted with the referents of the primitive descriptive terms of the language employed in ontological description. I assume for the moment that the two formulations are equivalent, and I will return to the second in the last section of this paper ${ }^{2}$.

Consider now a green spot in the visual field and assume that we are acquainted with it. In Bergmann's ontology the spot is a fact, namely, it is not a simple entity. It is assayed as a bare particular (an entity which has no nature whatsoever) exemplifying one character or universal. So, in order to hold that we are acquainted with the ontological simples, it seems that we must grant that if one is acquainted with the spot, then he is acquainted with its constituents too. Leaving aside the issue of being acquainted with universals for the moment, it remains to show that we are somehow presented with bare particulars.

It has been doubted that we can ever be acquainted with something bare. The reason is quite obvious: bare particulars lack natures, so we would not be able to answer the question: "What are you knowing when you say you are presented with a bare particular?". A plausible answer could be: "I'm not acquainted with any descriptive content, of course, but I'm acquainted with particularity itself". The argument runs as follows ${ }^{3}$ : consider two spots of the same colour, size, shape, and so on. Everyone will agree that, while we are seeing the two individual objects, we see both their qualitative sameness and their numerical difference. Given that universals can account only for qualitative aspects, we must acknowledge the existence of bare particulars in order to explain why we are acquainted with the difference between the two spots. For, if it would not be so, how can we distinguish the two indiscernible objects?

${ }^{1}$ Cf. Allaire, 1963; Chappell, 1964; Clatterbaugh, 1965; Allaire, 1965a and 1965b; Hochberg, 1965a and 1965b; Barber, 1966; Hochberg, 1966a and 1966b; Addis, 1967; Baker, 1967; Wilson, 1969; Grossman, 1974.

${ }^{2}$ It is not hard to find evidence for both of them in Bergmann's works. Cf. Bergmann, 1947a, pp. 185-186; Bergmann, 1958a, pp. 126-127; Bergmann, 1960a, pp. 45-46; Bergmann, 1960b, p. 174 and pp. 185-186; Bergmann, 1961, pp. 117-118; Bergmann, 1964 c, p. 307.

${ }^{3}$ Cf. Allaire, 1963, pp. 6-8. Hochberg also recalls this reasoning, in order to criticize it: Hochberg, 1965, pp. 124-126. 
Such a reasoning can be challenged in this way. We can grant both that we are presented with two objects which differ only numerically and that we are presented with their difference. We may ask, however, whether it follows from it that we are presented even with the ontological ground of their difference. Bare particulars, in other words, are supposed to be the elements "in" the spots which account for their difference, but the difference we are acquainted with is primarily that of the spots, not that of bare particulars directly. In other words, we begin with the numerical difference between the two spots: this is what we are acquainted with and what needs to be explicated. The realist ontologist then describes this state of affairs by having recourse to bare particulars and characters. Other ontologists, however, can advance alternative analyses in terms of perfect particulars, for instance, or universals together with coordinate qualities. Everyone of these proposals account for the difference between the two spots by means of different ontological devices. And, if all the accounts are adequate, then it seems that we cannot prefer one ontological analysis to the other by appealing to what we are presented with ${ }^{4}$. I found three possible replies to this piece of argument.

(A) The realist can charge his opponents by holding that their analyses are not completely successful. He maintains that only the explanation based on universals and bare particulars correctly accounts for the difference between the two indiscernible spots. An ontology of perfect particulars, for instance, cannot fully account for the sameness between the two spots, while the ontologist which employs coordinate qualities is not in a better position than the realist, because maintaining that we are acquainted with a coordinate quality seems to be as much difficult as holding that we are acquainted with a bare particular. Therefore the realist's ontological account should be preferred with respect to the others ${ }^{5}$.

It must be noticed, however, that if we are in agreement with this reasoning then the commitment to bare particulars seems to be grounded on dialectical reasons about their accounting for individuation, and not on the principle of acquaintance alone. We have not shown that we are acquainted with bare particulars; we have just shown that they are the easiest way to explain what we are presented with. Moreover, as we will see in the second part of this paper, there seem to be some problem with the bare

${ }^{4}$ References to these points can be found in Clatterbaugh, 1965, pp. 273-276 and in Hochberg, 1966, pp. 50-52.

${ }^{5}$ Cf. Addis, 1967; cf. also Oaklander, 1977, pp. 488-489. 
particulars theory of individuation. So neither the dialectical reasons could be satisfactory at the end.

(B) Alternatively, one can hold that the whole reasoning above is misleading, because it establishes a difference which is not really there. We said that we start from being acquainted with the spot, and then we advance an ontological analysis of it. One could observe, however, that if an analysis has to be taken seriously then what has been analysed should not be something different from the result of the analysis. It is not true that there are, so to speak, first the spot (which is one entity), and then the analysed spot (namely, several entities). For Bergmann the spot simply is the bare particular exemplifying the universal, and not a further entity. So, while we are presented with the spot we are acquainted with two distinct elements: a bare particular and a character. To claim that we are acquainted with the spot and not with its constituents is to confuse the unity of the fact with its alleged simplicity.

The source of the confusion seems to be the interference between the principle of acquaintance and another fundamental principle ruling over bare particulars, namely the Principle of Exemplification. It says that particulars are never presented unqualitied and qualities are never presented unexemplified $^{6}$. So it is true that, since bare particulars and universals always go together, one cannot be acquainted with one without being acquainted with the other. But this does not mean that you are not presented with them as such. An example may help: imagine you are the referee of a dancing competition for couples. Then you will always have in front of you a man and a woman dancing together. Even though the rules of the competition don't allow a person dancing alone, what you are presented with are two distinct human beings and not, so to speak, a man and a woman melted together.

Does this reply look convincing? It seems to me that, even if we grant that in being acquainted with a fact we are acquainted simply with its constituents' being together, a difficulty remains. One of Bergmann's main lessons is that a fact is not the same as the collection of its constituents ${ }^{7}$. Assume, for the sake of the argument, that we can be acquainted with each of its constituents separately: we will not be acquainted with the fact too. The difference between the fact $a \varepsilon \mathrm{Gr}$ and the collection $\{a, \mathrm{Gr}, \varepsilon\}$ is that in the first case the nexus of exemplification actually ties the bare particular

${ }^{6}$ This is stated by Bergmann many times. Cf., for instance, Bergmann, 1962a, pp. 7374; Bergmann, 1964b, pp. 277-278; Bergmann, 1967, p. 88.

${ }^{7}$ Cf., for instance, Bergmann, 1967, pp. 9-11, 22, 36. 
and the character together; in the second case it does nothing. So we must concede that, in being acquainted with the fact, we are acquainted with something more than its constituents. Or, to put it differently, we must concede that one can be acquainted with the constituents of a fact only if they are actually "in" the fact. Consequently, it seems that there is a sense according to which the spot has some priority with respect to the elements of its ontological analysis.

(C) A further argument for maintaining that bare particulars are presented is the following. Consider Bergmann's claim that bare particulars, at least in the case of spots in the visual field, must be conceived of as areas ${ }^{8}$. Then it seems hard to deny that, while a spot is presented to us, we are not acquainted with its area as well. Hence we are acquainted with bare particulars after all.

The reasoning of course is grounded in the claim that areas are particulars rather than properties. So we must try to understand why Bergmann was thinking so. For it does not seem immediately wrong to say, on the contrary, that the spot has an area in the same way it has a shape, a size, a colour, and so on. In the essay "Synthetic $A$ Priori" Bergmann advanced two replies to this objection". One involves the problem of individuation: Bergmann maintains that we cannot account for the difference between two spots which agree in all non-relational respects, if areas are included in them. I will deal with this problem in the next section of the paper, so here I face the other challenge only.

If I understand Bergmann correctly, he is saying that a spot in the visual field does not have an area in the same sense in which it has a shape and a size. The spot rather is an area which has, or exemplifies, a shape, a size, a colour, and so on. This means that, unlike having a shape and a size, being spatially extended (which I assume is synonymous to being an area) is not a further spatial property of a thing. In other words, it means that there is not a substratum, an "I don't know what", that has the properties of being extended, having a shape and having a size ${ }^{10}$.

So far so good, but after that Bergmann says both that he knows that an entity is an area because it has a shape and that to be an area is just to be an entity that has a shape. This sounds to me like the claim that to say of something that is spatially extended is to say merely that it has a shape. If the former analysis of Bergmann's text is correct, however, then it seems

\footnotetext{
${ }^{8}$ Cf. Bergmann, 1964b, p. 288; Bergmann, 1967, p. 74.

${ }^{9}$ Cf. Bergmann, 1964b, pp. 289-291.

${ }^{10}$ Cf. Wolterstorff, 1970, pp. 124-125; Grossmann, 1974, pp. 98-100.
} 
to me that one could find an easy reply to the argument for the acquaintance with bare particulars. It runs as follows: if being spatially extended is nothing but having a shape, then when we are acquainted with the area of the spot we are acquainted with nothing but the spot's shape. Shape, on the other hand, is a character. So at the end we are acquainted only with one of the spot's characters and not with its bare particular.

\section{Bare particulars and individuation}

One further reason for including bare particulars in someone's ontology is that they are supposed to ground individuation ${ }^{11}$. «A bare particular», says Bergmann in Realism «is a mere individuator. Structurally that is its only job. It does nothing else ${ }^{12}$. Consider two very simple objects, for instance, two green spots in the visual field. Assume that both of them are green and round, and that they have the same shape, size and so on. Briefly, we are presented with two objects which share all their non-relational properties. How then can an ontologist account for the difference between the two spots? It shall be quite intuitive to make appeal to the places of the spots, that is, to say that objects are singled out by means of their spatial properties. If spatial properties are regarded as relations, however, this task cannot be achieved, since relations presuppose their relata.

Thus it seems that our ontologist must introduce a constituent other than the ordinary properties of the spots (Green, Round and so on) in order to account for their difference. At this point two alternative options stand before him. The first is the so called theory of coordinate qualities. They are special properties of the spots which account for their position in the visual field and so for their individuation. "The second way of solving the problem of individuation», said Bergmann,

is to make the further constituent a bare particular. This notion [...] has two parts. Bare particulars neither are nor have natures. Any two of them, therefore, are not intrinsically but only numerically different. That is their bareness. It is impossible for a bare particular to be "in" more than one ordinary thing. That is their particularity. (Bergmann, 1967, p. 24)

${ }^{11}$ Though this debate was mostly contemporary to Bergmann (cf. Sellars, 1952 and Alston, 1954, as well as texts cited in footnote 1) it has some more recent occurrences as well: cf. Moreland, 1998; Mertz, 2001; Moreland - Pickavance, 2003.

${ }^{12}$ Bergmann, 1967, p. 25. Cf. also Bergmann, 1962b, pp. $92-93$. 
As is well known, Bergmann claimed that he preferred this option to the former because it provides a deeper ontological ground for individuation. That is because with the latter solution individuality relies on the connection between a bare particular and a universal, that is, between entities belonging to different ontological kinds tied together by the inhomogeneous nexus of exemplification. Coordinate qualities, on the other hand, are qualities among qualities. So, if they are linked to the other properties of the objects by something, it will be a thing like an a priori law that presumably will be dependent on their natures. While the first solution accounts for individuation by means of the relationship between different ontological categories, the second accounts for it by means of a homogeneous connection, and for Bergmann the first connection is indeed stronger than the second ${ }^{13}$.

In my opinion, however, grounding individuation only on the categorical level can lead to a couple of paradoxes which, in turn, may cast some doubts on the real advantages of employing bare particulars in order to solve the problem of individuation. Let me show what these paradoxes are. Assume, for the sake of the argument, that bare particulars really do their job and consider two spots, the first of which is white and square and the second oval and blue. Despite their difference in properties, they are different because of the two bare particulars in them:

$a \varepsilon(\mathrm{W} \& \mathrm{~S}) ; b \varepsilon(\mathrm{O} \& \mathrm{~B})$.

At this point, however, what prevents us from assaying the same circumstance as a single bare particular exemplifying all the four characters? This case seems to be impossible because we cannot conceive of an object which is both white and blue, and both square and oval. This answer can't be accepted, however. For it makes the individuation of the two spots dependent on the a priori law which says that no ordinary object can have at the same time more than one shape or more than one colour. On the contrary, we are under the assumption that only bare particulars provide for individuation. Thus, only formation rules are pertinent for the categorical level. Since the formula

\section{$a \varepsilon(\mathrm{W} \& \mathrm{~S} \& \mathrm{O} \& \mathrm{~B})$}

does not seem ill-formed in Bergmann's jargon, it should be accepted. Yet it describes a state of affair pretty different than before: there we had two facts, here we count only one.

${ }^{13}$ Bergmann, 1967, pp. 24-28, 46-48. Cf. also Hausman, 1967, pp. 41, 45-46. 
If solving the problem of individuation means to be able to count how many things of a certain kind there are, then it seems that we are getting in trouble. The reason is quite obvious: what has been done with the four characters can in principle be carried on for all the characters we can find in our world. So we could have, paradoxically, our world actually composed by a single bare particular exemplifying a very long conjunction of characters. Different remarks come to my mind, at the moment.

(A) Firstly, I guess that Bergmann would not rule out the case of a single bare particular exemplifying all the simple characters of the world, at least in principle. It would correspond, in his mind, to count the whole visual field as one individual. That indeed seems to be possible, although it is never the case in practice. The fundamental point is how to assay the parts of the field. In our example, since there is a single bare particular exemplifying all the four characters, we can say that there is only one object, and we can regard the two distinct spots as parts of it. In other words, we would have a strange object with a part which is white and square and a part which is oval and blue. According to Bergmann, however, there is also a bare particular for each of the two spots, and these bare particulars are in the relation part-whole with the bare particular exemplifying the whole visual field.

This assay depends on Bergmann's peculiar explanation of the partwhole relation we can find in chapter IV of Realism ${ }^{14}$. For Bergmann, partwhole is not a relation between objects, it is a relation between bare particulars. So it seems that the paradox arises only from my wrong attempt to single out "parts" of the visual field by means of a priori rules between qualities. If, on the contrary, their individuation is grounded on bare particulars too, then there is not a paradox at all. It remains a doubt, however. Bergmann says both that the part-whole relation holds between bare par-ticulars and that its ground is an a priori truth about space. But if it is so, then it seems to me that the individuation of parts by means of bare particulars is subordinated to the nature of the part-whole relation which, in turn, seems to be grounded on the a priori level rather than on the categorical level.

(B) My second remark is the following: after all, the paradox arose because we were allowed to bind universals together on the right side of the formula, thus writing

$$
a \varepsilon(\mathrm{W} \& \mathrm{~S} \& \mathrm{O} \& \mathrm{~B})
$$

${ }^{14}$ Bergmann, 1967, pp. 71-82. Further reflections about the part-whole relation can be found in Bergmann, 1958c, pp. 227-230; Bergmann, 1964b, pp. 287-290, 296-330. 
instead of

$$
a \varepsilon(\mathrm{W} \& \mathrm{~S}) ; b \varepsilon(\mathrm{O} \& \mathrm{~B})
$$

If one rejects this possibility, however, it seems to me that he must reject also the writing

$$
a \varepsilon(\mathrm{W} \& \mathrm{~S}) ; b \varepsilon(\mathrm{O} \& \mathrm{~B})
$$

and say that the only correct description is

$$
a \varepsilon \mathrm{W} \wedge a \varepsilon \mathrm{S} ; b \varepsilon \mathrm{O} \wedge b \varepsilon \mathrm{B}^{15},
$$

which amounts to say that each bare particular exemplifies every simple character independently, and that it is the presence of the numerically same bare particular in the different atomic facts which grounds the individuation of the two spots.

This is fine, but not completely satisfactory. For, if a bare particular exemplifies every simple character separately, one could think that it is just an arbitrary circumstance that the same bare particular co-exemplifies different characters. In other words, it seems that nothing prevents us from delivering a different bare particular to every single simple character, thus having:

$c \varepsilon \mathrm{W} \wedge d \varepsilon \mathrm{S} \wedge e \varepsilon \mathrm{O} \wedge f \varepsilon \mathrm{B}$.

At this point the notion of bare particular turns out to be redundant. Insofar as simple characters are distinguished as such by their natures, there is no need to introduce a bare particular to say that white is different from blue. Of course, bare particulars still serve to account for the concreteness of characters: here there are four instantiated simple universals and not four universals alone. It seems to me, however, that the danger of nominalism has never been as close as in this situation: just change each atomic fact with a perfect particular and say that our object is merely a conjunction of them, and the alleged advantage of accepting bare particulars vanishes.

The second paradox remains to be shown: it can be stated as follows. Recall the two indiscernible spots, and assume that the ground of their difference is their bare particulars, which are only numerically different. Then the two spots need not be in different places: they can even be exactly superimposed. Two different bare particulars, in the present case, would exemplify the same universals, including spatial relations. We will no more "see", so to speak, the two green and round spots: we will see the same

${ }^{15}$ Even though it is not particularly relevant here, I preserve the distinction between the sign " $\wedge$ ", which denotes the nexus of conjunction between facts, and the sign " $\&$ ", which denotes a pseudo-nexus connecting universals only. 
thing that we would see if the spot were one. Yet, how could we distinguish between the one-particular case and the two-particulars case? Since we are not acquainted with bare particulars independently, we cannot count how many of them stay behind the same spot's presentation. It could be one, it could be two, it could be any number, finite or infinite, of them.

The point at stake is the same as before: if solving the problem of individuation is to answer the question "How many things of a certain kind are there?", then bare particulars hardly help to single out objects. One can answer this question only if he has previously assumed that there are, say, two bare particulars (and so two objects), rather than one. This assumption, I must notice, is quite arbitrary. In other words: given a presentation of a character whatsoever, it seems that there is no reason to say that there is only one bare particular which is exemplifying it rather than two or more. Yet the number of facts involved is different, so it seems that to distinguish between the one-particular case and the two-particulars case has some ontological relevance.

One could reply that the case of two exactly superimposed objects is simply absurd. According to Bergmann, for example, bare particulars must be thought of as indivisible areas (or sectors of the visual field) which exemplify properties, so that the two alleged superimposed areas would actually be the same. Yet a perplexity remains. In "Synthetic A Priori" Bergmann says that if we regard areas as properties and allow objects to share all their properties including areas, then we would no longer be able to single out any two of them. So we must conceive of areas as bare particulars $^{16}$.

If areas are bare particulars, however, then what does prevent them from being superimposed while remaining distinct? We are no more under the assumption that areas are character-like entities: in such a case, two superimposed objects would be sharing literally the same character. Now, on the contrary, areas are bare particulars (i.e., numerically different) for hypothesis. So it seems to me that they can be superimposed without any particular problem. Obviously one can persist in denying that areas can be superimposed while remaining distinct, even if areas are regarded as bare particulars. But this looks more like an a priori truth about space than a categorical demand of someone's ontology.

${ }^{16}$ Cf. Bergmann, 1964b, p. 289. 


\section{Bare particulars and ideal language}

Together with the above mentioned reasons for bringing bare particulars in someone's ontology, I found in Bergmann's works a further and stronger motivation to accept them. Bergmann repeatedly states, especially in the papers belonging to The Metaphysics of Logical Positivism, that in ontology he applies the ideal language method ${ }^{17}$. The basic idea is that of a formalism which, when interpreted in a suitable way, expresses the ontological constitution of the world. It is not difficult then to find a very accurate correspondence between Bergmann's favourite formalism, namely the unproblematic parts of Principia Mathematica, and the elements of his ontology.

The division between logical and descriptive terms is mirrored by the distinction between subsistents and existents, which are, respectively, the form and the matter of the world. All primitive descriptive terms are names, and the remaining can be defined by means of them. Individual constants, written by lower case letters, stand for particulars. Upper case letters are predicative constants, which name universals and relations. The simple clause, expressed by the juxtaposition of these kinds of signs, has its counterpart in the fundamental nexus of exemplification, which gives raise to the atomic fact. Thus, 'This is green' both denotes a fact and is a well-formed sentence, while 'Green is this' means nothing since it corresponds to an ill-formed sentence. Just as complex sentences are built from simple ones by means of the standard connectives, so are complex facts with respect to atomic ones ${ }^{18}$. Even quantifiers and variables have, at least if we agree with what Bergmann claimed in the essay "Generality and Existence", an ontological counterpart we are acquainted with ${ }^{19}$.

${ }^{17}$ I am making reference especially to the following papers: Bergmann, 1946; 1947b; $1950 ; 1952 ; 1953 ; 1954 b$. Later works also contain mentions of the ideal language: $c f$. Bergmann, 1960a, pp. 48-49; 1960b, p. 172; 1962a and 1962b.

${ }^{18}$ Of course connectives (at least conjunction and disjunction), being represented by signs of the ideal language, denote true constituents of the world: cf. Bergmann, 1963, p. 130.

19 «Each quantifier represents something which is sometimes presented. Had it never been presented, we would not know what the quantifier meant [...]. Generality and existence subsist. That is the thesis» (Bergmann, 1962a, p. 70). «A form 3 is a geometrical character exemplified by sentences; the feature of the world's form it represents is shared (not: exemplified!) by the facts these sentences express. Each form $_{3}$ is associated with a schema. Each instance of the schema exemplifies the form ${ }_{3}$. Take ' $f(x)$ ', the simplest of all schemata. The form 3 with which it is associated is 
Thus, if ontology abides by such a biunivocal correspondence between the ontological kinds and the signs of the ideal language, it seems almost unavoidable to acknowledge the existence of bare particulars. We need something which corresponds to the primitive descriptive terms of zerotype. Since the same predicative term can be juxtaposed to any number of them, while they are not predicated of anything, they will be, ontologically speaking, particulars. Since only primitive descriptive terms of type one and higher are supposed to denote universals and relations, there is no nature left to be expressed by the zero-type terms, so their referents will be bare. The only relevant role of bare particulars, in other words, is to correspond on the ontological side to the individual constants of the ideal language. Other jobs, like individuation or accounting for being presented with particularity, are secondary and, as we have seen before, somewhat problematic.

Moreover, this biunivocal correspondence can even explain why such problems arise. Consider acquaintance: the trouble was that we cannot distinguish between being acquainted with the particularity of the spot and with bare particulars. Since we are acquainted with spots, the existence of bare particulars can only be inferred from the particularity of the spot and not be experienced as such. All of this follows from the formation rules of the ideal language. They require that the simple clause is always formed by juxtaposition of a predicative and an individual term. The ontological meaning of this is that bare particulars are always exemplifying universals, so that they can never be presented alone.

For what concerns individuation, paradoxes were born from splitting the thisness aspect of individuation and the suchness aspect, and from claiming that only the first is relevant to single out objects. The split is due to the distinction, in the ideal language, between predicative and individual terms. The one-particular world and the two superimposed spots can be conceived because in the ideal language there aren't rules which prevent them from being mentioned by well-formed sentences. More concisely: ordinary objects are usually singled out by means of laws such as 'no

exemplified by ' $G(a)$ ', ' $F(b)$ ', and so on. The feature this form ${ }_{3}$ represents is shared by all and only those facts which consist of an individual exemplifying a character. Form $_{3}$, being a geometrical character, consists, in the sense explicated, of form 1 and form $_{2}[\ldots]$. Having distinguished between form 1 , form 2 , and form 3 , we have as it were distinguished three regions in the realm of subsistence, each containing what is expressed by one of the three geometrical (syntactical) "forms") (Bergmann, 1962a, pp. 82-83). 
single object may be in two place at one time', 'no single object can have two shapes at one time', and so on. These laws stand, from Bergmann's point of view, on the a priori level which rules the natures of the characters rather than on the categorical level of well- and ill-formed sentences. So, having placed individuation on the categorical level alone, Bergmann simply has no tools to account for the individuation of ordinary objects ${ }^{20}$.

My last remark is the following. Assume that Bergmann's point is simply what has been shown above, i.e., that we must concede the existence of bare particulars in order to give ontological meaning to a formalism like that of Principia Mathematica. One could reply, of course, that what has been advanced is just a semantic proposal. So, either the same formalism can be interpreted in another way, or a different formalism can be provided and chosen as our official ideal language. In both cases we could somehow avoid to commit ourselves to bare particulars.

It seems to me that a large part of Bergmann's philosophy can be seen as devoted to put these claims to shame. He spent a lot of time to show that both different readings and alternative formalisms dismiss the ontological ground of something which, on the contrary, was perspicuously shown by his interpretation. With his remarks against Frege, for instance, Bergmann was challenging an interpretation of the dependence between predicative and individual terms according to which predicative constants are seen as something which need to be saturated in order to gain ontological meaning $^{21}$. With his criticism of Quine, Bergmann was, among other things, showing the troubles of a formalism which has no constants and only a kind of variables ${ }^{22}$. In his long analysis of Goodman's writings, finally, Bergmann was dealing with the disappointing ontological consequences of using a different formalism as the ideal language ${ }^{23}$.

Since I am not sufficiently familiar with the works of Bergmann's opponents, I am not in a position to judge whether he is truly the winner of all

${ }^{20}$ I share this conclusion with Oaklander, whose essay was for me very instructive: cf. Oaklander, 1977, especially pp. 482-487. For a reply by Bergmann, cf. Bergmann, 1977.

${ }^{21}$ Cf. Bergmann, 1958b; 1956 and 1963 also have some relevance here.

${ }^{22}$ Cf. Bergmann, 1950; 1953; 1954c. On these topics cf. also Hochberg, 1957a and $1957 b$.

${ }^{23}$ Cf. Bergmann, 1967, pp. 12-17, 38-39, 44-45. Cf. also Bergmann, 1952, pp. 115116; Bergmann, 1954c, pp. 131-132. For what concerns Bergmann's opponents, readers can see Goodman, 1951 and 1956. Moreover, Hausman, 1967 contains a reading of Goodman's ontology from a Bergmannian point of view (notice that this work appeared the same year as Realism). 
these matches. If Bergmann's criticisms are correct, on the other side, it seems that we should agree with his interpretation of the formalism and so to commit ourselves to bare particulars. At least as long as a further ideal language which can depart from them is found.

\section{Francesco Martinello}

Università di Torino

francesco.martinello@unito.it

\section{REFERENCES}

Addis, L. (1967), "Particulars and Acquaintance", Philosophy of Science, XXIV, pp. 251-259.

Allaire, E.B. (1963), "Bare Particulars", Philosophical Studies, XIV, pp. 1-8.

- (1965a), "Another Look at Bare Particulars", Philosophical Studies, XVI, pp. 1521.

- (1965b), "Ontology and Acquaintance: A Reply to Clatterbaugh", Philosophy of Science, XXXII, pp. 277-280.

Alston, W.P. (1954), "Particulars - Bare and Qualified", Philosophy and Phenomenological Research, XV, pp. 253-258.

Baker, R. (1967), "Particulars: Bare, Naked, and Nude", Noûs, I, pp. 211-212.

Barber, K. (1966), "Bare Particulars and Acquaintance”, Dialogue, V, pp. 580-583.

Bergmann, G. (1946), "Remarks on Realism”, in Bergmann (1954a), pp. 78-105.

- (1947a), "Sense Data, Linguistic Conventions, and Existence", in Bergmann (1954a), pp. 176-196.

— (1947b), "Russell on Particulars", in Bergmann (1954a), pp. 197-214.

- (1950), "A Note on Ontology", in Bergmann (1954a), pp. 238-242.

- (1952), "Two Types of Linguistic Philosophy”, in Bergmann (1954a), pp. 106-131.

- (1954a), The Metaphysics of Logical Positivism, Longmans, Green \& Co, New York; $2^{\text {nd }}$ ed. University of Wisconsin Press, Madison (WI), 1967.

- (1954b), "Bodies, Minds, and Acts", in Bergmann (1954a), pp. 132-152.

- (1954c), "Particularity and the New Nominalism", in Bergmann (1959), pp. 91-105.

- (1956), "Propositional Functions", Analysis, XVII, pp. 43-48.

— (1958a), "Individuals", in Bergmann (1959), pp. 124-131.

— (1958b), "Frege's Hidden Nominalism", in Bergmann (1959), pp. 205-224.

- (1958c), "Some Reflections on Time", in Bergmann (1959), pp. 225-263.

- (1959), Meaning and Existence, University of Wisconsin Press, Madison (WI). 
- (1960a), "Ineffability, Ontology, and Method", in Bergmann (1964a), pp. 45-63.

- (1960b), "Strawson's Ontology", in Bergmann (1964a), pp. 171-192.

- (1961), "Physics and Ontology", in Bergmann (1964a), pp. 108-123.

— (1962a),"Generality and Existence", in Bergmann (1964a), pp. 64-84.

— (1962b), "Meaning”, in Bergmann (1964a), pp. 85-97.

— (1963), “Ontological Alternatives”, in Bergmann (1964a), pp. 124-157.

- (1964a), Logic and Reality, University of Wisconsin Press, Madison (WI).

— (1964b), "Synthetic A Priori”, in Bergmann (1964a), pp. 272-301.

— (1964c), "Realistic Postscript", in Bergmann (1964a), pp. 302-340.

- (1967), Realism. A Critique of Brentano and Meinong, University of Wisconsin Press, Madison (WI).

- (1977), "Some Comments on Professor Oaklander's 'Particulars, Positional Qualities, and Individuation"”, Philosophy of Science, XLIV, pp. 491-493.

Chappell, V.C. (1964), "Particulars Re-Clothed”, Philosophical Studies, XV, pp. 6064.

Clatterbaugh, K.C. (1965), "General Ontology and the Principle of Acquaintance", Philosophy of Science, XXII, pp. 272-276.

Goodman, N. (1951), The Structure of Appearance, Harvard University Press, Cambridge (MA).

— (1956), "A World of Individuals", in P. Benacerraf - H. Putnam, eds., Philosophy of Mathematics, Blackwell, Oxford, pp. 197-210.

Grossmann, R. (1974), "Bergmann's Ontology and the Principle of Acquaintance", in M.S. Gram - E.D. Klemke, eds., The Ontological Turn. Studies in the Philosophy of Gustav Bergmann, University of Iowa Press, Iowa City (IO), pp. 89-113.

Hausman, A. (1967), "Goodman's Ontology", in A. Hausman - F. Wilson, Carnap and Goodman: Two Formalists, Martinus Nijhoff, The Hague, pp. 1-95.

Hochberg, H. (1957a), "Professor Quine, Pegasus, and Dr. Cartwright", Philosophy of Science, XXIV, pp. 191-203.

— (1957b), “On Pegasizing”, Philosophy and Phenomenological Research, XVII, pp. 551-554.

— (1965a), “On Being and Being Presented”, Philosophy of Science, XXXII, pp. 123136.

- (1965b), "Universals, Particulars, and Predication", Review of Metaphysics, XIX, pp. 87-102.

- (1966a), "Ontology and Acquaintance”, Philosophical Studies, XVII, pp. 49-55.

- (1966b), "Things and Descriptions", American Philosophical Quarterly, III, pp. 19.

Mertz, D.W. (2001), "Individuation and Instance in Ontology", Australasian Journal of Philosophy, LXXIX, pp. 45-61. 
Moreland, J.P. (1998), "Theories of Individuation: a Reconsideration of Bare Particulars", Pacific Philosophical Quarterly, LXXIX, pp. 251-263.

Moreland, J.P. - Pickavance, T. (2003), "Bare Particulars and Individuation. Reply to Mertz", Australasian Journal of Philosophy, LXXXI, pp. 1-13.

Oaklander, L.N. (1977), "Particulars, Positional Qualities and Individuation”, Philosophy of Science, XLIV, pp. 478-489.

Sellars, W. (1952), "Particulars", Philosophy and Phenomenological Research, XIII, pp. 184-199.

Wilson, F. (1969), "The Role of a Principle of Acquaintance in Ontology", The Modern Schoolman, XLVII, pp. 37-56.

Wolterstorff, N. (1970), “Bergmann's Constituent Ontology”, Noûs, IV, pp. 109-134. 